\title{
ON THE EXISTENCE OF LONG PERIODIC ORBITS NEAR THE LIAPUNOV'S PERIODIC FAMILY IN GENERAL REASONANCE CASES
}

\author{
By HideKazU ITo
}

\section{Introduction.}

In this note, we are concerned with the existence of periodic solutions for a Hamiltonian system with $n$ degrees of freedom

$$
\frac{d x_{k}}{d t}=\frac{\partial H}{\partial y_{k}}, \quad \frac{d y_{k}}{d t}=-\frac{\partial H}{\partial x_{k}} \quad(k=1, \cdots, n) .
$$

We assume that the Hamiltonian function $H(x, y)=H\left(x_{1}, \cdots, x_{n}, y_{1}, \cdots, y_{n}\right)$ is smooth near the origin and vanishes with its first-order derivatives at the origin, which implies the origin is an equilibrium point. Here and in what follows, "smooth" means always $C^{\infty}$. It is to be noted that the Hamiltonian function $H(x, y)$ is an integral for the system (1.1), i. e., the function $H(x, y)$ is constant along a solution curve for (1.1).

Let $S$ denote the Hessian matrix of $H(x, y)$ at the origin and

$$
J=\left[\begin{array}{rr}
0 & I \\
-I & 0
\end{array}\right],
$$

where $I$ is the $n \times n$ identity matrix. Then $J S$ is the coefficient matrix of the linear terms of the vector field of (1.1) about the origin. As is well known [12], the eigenvalues of $J S$ occur in pairs $\pm \lambda_{1}, \cdots, \pm \lambda_{n}$. If $\lambda_{1}$ is purely imaginary and none of the $n-1$ quotients $\lambda_{k} / \lambda_{1}(k=2, \cdots, n)$ is an integer, a well-known theorem by Liapunov guarantees the existence of a one-parameter family of periodic solutions near the equilibrium (see [10] or section 16 of [12]). Recently many researches have been devoted to the study of the existence of periodic solutions in the cases when there exist integer-multiple eigenvalues of $\lambda_{1}$ among $\lambda_{2}, \cdots, \lambda_{n}$, i, e., resonance cases. In our previous paper [7], we considered an autonomous system possessing a nondegenerate integral under general resonance cases and established an existence theorem for long periodic solutions near an equilibrium. In this note, we restrict ourselves to the Hamiltonian system (1.1) and consider the same general resonance situation as in [7]. We then assume

Received December 27, 1980 
that the eigenvalues $\pm \lambda_{k}$ of $J S$ satisfy the following conditions for an integer $r(\geqq 2)$ :

[A.1] $\pm \lambda_{k}= \pm \imath \sigma_{k}(k=1, \cdots, r)$ are $r$ pairs of purely imaginary eigenvalues all of which are integer multiples of $\lambda_{1}=i \sigma_{1}$ such that $\left|\sigma_{1}\right|<\left|\sigma_{2}\right|$ $<\cdots<\left|\sigma_{r}\right|$ and

$$
\sum_{k=1}^{r} \jmath_{k} \sigma_{k} \neq 0
$$

for all integer valued vectors $\left(j_{1}, \cdots, \jmath_{r}\right)$ with $1 \leqq \sum_{k=1}^{r}\left|\jmath_{k}\right| \leqq 4$.

[A.2] no $\pm \lambda_{k+r}(k=1, \cdots, s)$ is an integer multiple of $\lambda_{1}$, where $s=n-r$.

Under the assumptions [A.1] and [A.2], the Liapunov's theorem guarantees the existence of a one-parameter family of periodic solutions near the equilibrium whose primitive (minimal) periods are close to $2 \pi /\left|\sigma_{r}\right|$. The previous paper dealt with the existence of long periodic solutions near the equilibrium, and we know a sufficient condition for the existence of periodic solutions near the origin with primitive periods near $2 \pi /\left|\sigma_{1}\right|$ under [A.1] and [A.2], which are "long" periodic solutions in contrast with the periodic solutions given by the Liapunov's theorem. The aim of this note is to establish the existence of periodic orbits whose primitive periods are close to $2 \pi /\left|\sigma_{1}\right|$ near the above Liapunov's periodic family. Our sufficient condition for the existence of the long periodic orbits will be stated in terms of the coefficients of the second and fourth order terms of a normal form of the Hamiltonian function. It is stated in the next section and the proof is given in section 3. K. R. Meyer and J.I. Palmore [8] have obtained the similar result for Hamiltonian systems with two degrees of freedom. To establish the existence of periodic orbits as that of fixed points for some mapping (Poincaré mapping), they obtained an area-preserving mapping. On the other hand, we shall obtain an exact-canonical mapping in place of an area-preserving one. To prove the existence of fixed points for it, we use the argument due to G.D. Birkhoff ([3], [4]) and Poincaré (see [1] Appendix 9). Furthermore, a generalization of the theorem is given in section 4 , where we also discuss the connection between the results in this paper and our previous one [7].

As for the existence of periodic orbits near a given periodic motion, G. D. Birkhoff [3] studied a fixed point theorem, which is a generalization of Poincaré's geometric theorem (see [2]), and G.D. Birkhoff and D.C. Lewis [4] applied it for establishing the existence of periodic motions of Hamiltonian systems near a given periodic motion. This is called the Birkhoff-Lewis fixed point theorem. It was improved by T.C. Harris [6], and its most generalized form is found in J. Moser [9]. These are interpreted as fixed point theorems for a local symplectic diffeomorphism near a fixed point, and their application to the Poincaré mapping around a given periodic motion guarantees the existence of infinitely many periodic orbits with sufficiently long periods. The basic idea for proving the existence of fixed (periodic) points is due to G.D. Birkhoff, whose argument 
is originally due to Poincaré's one (see [1] Appendix). It is the reduction of the desired fixed points to critical points for a certain function. Our way of research depends on the similar argument, and we consider the Poincaré mappings around the periodic orbits given by the Liapunov's theorem. But we specialize the discussion to the resonance situation defined by [A.1] and [A.2], and establish the existence of long periodic orbits in the above sense, not "sufficiently long" periodic orbits. In the proof, a stretching transformation will play an important role.

I would like to express my sincere gratitude to Professor T. Nishimoto and Professor Y. Hirasawa for their constant encouragement and valuable advices. I would like to thank Dr. H. Yamada for several helpful discussions and valuable comments.

\section{Statement.}

In order to formulate our result, we have to prepare a normal form of the Hamiltonian function. This is presented by (2.1) in the following lemma, in which $\langle\cdot, \cdot\rangle$ denotes the usual scalar product.

LEMMA 1. Consider the Hamiltonan system (1.1) near the origin. Let $S$ be the Hessian matrix of $H(x, y)$ at the orrgin. Assume that the ergenvalues of JS satisfy [A.1] and [A.2]. Then there exists a real analytic canonical transformation of variables

$$
(x, y) \longrightarrow(u, \xi, v, \eta)=\left(u_{1}, \cdots, u_{r}, \xi_{1}, \cdots, \xi_{s}, v_{1}, \cdots v_{r}, \eta_{1}, \cdots, \eta_{s}\right)
$$

such that the Hamiltonian function $H=H(x, y)$ becomes

$$
H=\sum_{k=1}^{r} \sigma_{k} \tau_{k}+\frac{1}{2} \sum_{k, l=1}^{r} q_{k l} \tau_{k} \tau_{l}+\frac{1}{2}\langle B \zeta, \zeta\rangle+K(u, v, \xi, \eta), \quad \tau_{k}=\frac{u_{k}^{2}+v_{k}^{2}}{2},
$$

where $\left(q_{k l}\right)$ is a real $r \times r$ symmetruc matrix and $B$ is a real $2 s \times 2 s$ symmetric matrix, and $K(u, v, \xi, \eta)$ is a smooth function near the origin such that

$$
K\left(u, v, \xi^{2}, \eta^{2}\right)=O\left((|w|+|\zeta|)^{5}\right) .
$$

Here $w$ (resp. $\zeta)$ is a column vector combined with $u$ and $v$ (resp. $\xi$ and $\eta$ ), and $\xi^{2}\left(\right.$ resp. $\left.\eta^{2}\right)$ is a column vector with entries $\xi_{k}^{2}$ (resp. $\left.\eta_{k}^{2} ; k=1, \cdots, s\right)$.

The transformation is said to be canonical (or symplectic) if it preserves the 2-form $\sum_{k=1}^{n} d x_{k} \wedge d y_{k}$, which implies the above transformation satisfies

$$
\sum_{k=1}^{n} d x_{k} \wedge d y_{k}=\sum_{k=1}^{r} d u_{k} \wedge d v_{k}+\sum_{k=1}^{s} d \xi_{k} \wedge d \eta_{k} .
$$

The system (1.1) is transformed into the Hamiltonian system defined by the Hamiltonian function (2.1), which can be written in the vector form 


$$
\frac{d w}{d t}=J_{r} \frac{\partial H}{\partial w}, \quad \frac{d \zeta}{d t}=J_{s} \frac{\partial H}{\partial \zeta},
$$

with

$$
J_{\nu}=\left[\begin{array}{rr}
0 & I_{\nu} \\
-I_{\nu} & 0
\end{array}\right] \quad(\nu=r, s),
$$

where $\partial H / \partial w$ and $\partial H / \partial \zeta$ are the vectors of first-order derivatives of $H(w, \zeta)$ given by (2.1), and $I_{\nu}$ is the $\nu \times \nu$ identity matrix.

The normal form (2.1) is used already by S. M. Graff [5] in a similar situation to [A.1] and [A.2]. The proof of this lemma is done by defining the desired canonical transformation by a generating function, which is determined by comparison of coefficients. However this can be done by following the argument in the Appendix of [5], and so we omit the proof of the lemma.

To state our result, we introduce an $(r+1) \times(r+1)$ matrix

$$
P=\left[\begin{array}{c|c|c}
\left(q_{k l}\right) & \sigma_{1} \\
& \sigma_{r} \\
\hline \sigma_{1} \cdots & \sigma_{r} & 0
\end{array}\right] .
$$

Further let us introduce $r \times r$ matrices

$$
\begin{aligned}
& \text { (k) } \\
& Q_{k}=\left[\begin{array}{ccccc}
q_{11} & \cdots & \sigma_{1} & \cdots & q_{1 r} \\
q_{21} & \cdots & \sigma_{2} & \cdots & q_{2 r} \\
\vdots & & \dot{y}_{r} & & : \\
q_{r 1} & \cdots & \sigma_{r} & \cdots & q_{r r}
\end{array}\right] \quad(k=1, \cdots, r),
\end{aligned}
$$

whose determinant is equal to the cofactor of the element $p_{r+1, k}=\sigma_{k}$ in the matix $P$. Then our result is stated as follows:

THEOREM A. Consider the Hamiltonan system (1.1) near the ortgin. Let the ergenvalues of JS satisfy [A.1] and [A.2]. Assume in the normal form (2.1) that

$$
\left(\sigma_{r}|P|\right) \cdot\left|Q_{k}\right|<0 \quad(k=1, \cdots, r),
$$

where $|\cdot|$ denotes the determinant of the matrix. Then, on each energy surface $H=\sigma_{r} \varepsilon^{2}$ with sufficiently small $\varepsilon>0$, there exist at least $r$ periodic orbits for (1.1) with primitive periods near $2 \pi /\left|\sigma_{1}\right|$ in a $O(\varepsilon)$-neighborhood of the periodic orbit belonging to the Liapunov's periodic family whose primitive period is close to $2 \pi /\left|\sigma_{r}\right|$.

\section{Proof.}

We will give the proof of the theorem in several steps.

i) The stretching transformation 
By Lemma 1, we may begin with the Hamiltonian system (2.3) with (2.1). If we introduce a stretching transformation

$$
w \longrightarrow \varepsilon w, \quad \zeta \longrightarrow \varepsilon^{2} \zeta,
$$

with a small positive parameter $\varepsilon$, then the Hamiltonian function (2.1) becomes

$$
\begin{aligned}
& H\left(\varepsilon w, \varepsilon^{2} \zeta\right)=\varepsilon^{2} \hat{H}(w, \zeta, \varepsilon), \\
& \hat{H}(w, \zeta, \varepsilon)=\sum_{k=1}^{r} \sigma_{k} \tau_{k}+\frac{\varepsilon^{2}}{2} \sum_{k, l=1}^{r} q_{k l} \tau_{k} \tau_{l}+\frac{\varepsilon^{2}}{2}\langle B \zeta, \zeta\rangle+O\left(\varepsilon^{3}\right),
\end{aligned}
$$

and the Hamiltonian system (2.3) is transformed into

$$
\frac{d w}{d t}=J_{r} \frac{\partial \hat{H}}{\partial w}, \quad \frac{d \zeta}{d t}=\varepsilon^{-2} J_{s} \frac{\partial \hat{H}}{\partial \zeta},
$$

The 2 -form (2.2) is taken into

$$
\varepsilon^{2} \sum_{k=1}^{r} d u_{k} \wedge d v_{k}+\varepsilon^{4} \sum_{k=1}^{s} d \xi_{k} \wedge d \eta_{k} .
$$

We will observe the Liapunov's periodic family under this stretching transformation.

LEMMA 2. Consider the system (2.3) with (2.1) near the orgin. Assume that the eigenvalues of the coefficient matrix of the linear terms of the vector field of (2.3) satısfy [A.1] and [A.2]. Then there exists a family of periodic solutions depending on a small parameter $\varepsilon>0$, with $\varepsilon=0$ corresponding to the origin, and whose primitive period is $2 \pi /\left|\sigma_{r}\right|+O\left(\varepsilon^{2}\right)$. Each periodic orbit belonging to this periodic family intersects transversally a submanifold $\Sigma$ of codimension 1 defined by

$$
\Sigma: v_{r}=0, \quad u_{r}>0,
$$

and its intersection with $\Sigma$ is given by

$$
\left\{\begin{array}{l}
u_{r}=\sqrt{2} \varepsilon, \quad v_{r}=0, \\
u_{k}=O\left(\varepsilon^{2}\right), \quad v_{k}=O\left(\varepsilon^{2}\right) \quad(k=1, \cdots, r-1), \quad \zeta=O\left(\varepsilon^{3}\right) .
\end{array}\right.
$$

Remarks. (i) The periodic family whose existence is guaranteed in this lemma is just the Liapunov's periodic family.

(ii) In (3.6), the relation $\mathrm{u}_{r}=\sqrt{2} \varepsilon$ is obtained by adjusting the $u_{r}$ such that $\tau_{r}=\varepsilon$ in $\Sigma$, and then it is possible to choose the parameter $\varepsilon$ so that $u_{r}=O(\varepsilon)$ in general in place of $u_{r}=\sqrt{2} \varepsilon$.

(iii) By (3.6), the energy surface where exists each periodic orbit belonging to this Liapunov's periodic family is given by the form

$$
H=\sigma_{r} \varepsilon^{2}+O\left(\varepsilon^{4}\right) .
$$


This shows that the energy surface $H=\sigma_{r} h$, with sufficiently small $h>0$, contains exactly one periodic orbit belonging to the Liapunov's periodic family.

(iv) The parameter $\varepsilon$ can be considered as the stretching parameter in (3.1). Then, after introducing the stretching transformation (3.1), we have

$$
\begin{cases}u_{r}=\sqrt{2}, & v_{r}=0 \\ u_{k}=O(\varepsilon), & v_{k}=O(\varepsilon) \quad(k=1, \cdots, r-1), \quad \zeta=O(\varepsilon) .\end{cases}
$$

Proof. This lemma is interpreted as the Liapunov's theorem for the system (2.3) with the Hamiltonian function of the special form (2.1), and then it is the special relation (3.6) that we wish to show. The proof is done by using the argument introduced by D.S. Schmidt [10].

Instead of (2.3), we consider the one-parameter system

$$
\frac{d w}{d t}=\left(J_{r}+\mu I_{r}\right) \frac{\partial H}{\partial w}, \quad \frac{d \zeta}{d t}=\left(J_{s}+\mu I_{s}\right) \frac{\partial H}{\partial \zeta},
$$

where $\mu$ is a real parameter. Since $H(w, \zeta)$ is an integral for (2.3), there exist no periodic solution with $\mu \neq 0$ for (3.9) such that the gradient vector of $H(w, \zeta)$ does not vanish identically along it. For this, see [10] (or refer to Lemma 4 in [7]).

Introducing a stretching transformation (3.1) together with a stretching of the parameter $\mu, \mu \rightarrow \varepsilon \mu$, the transformed system reads

$$
\frac{d w_{r}}{d t}=L(\mu, \varepsilon) w_{r}+O\left(\varepsilon^{2}\right), \quad \frac{d z}{d t}=M z+O(\varepsilon),
$$

where

$$
w_{r}=\left[\begin{array}{l}
u_{r} \\
v_{r}
\end{array}\right], \quad L(\mu, \varepsilon)=\left[\begin{array}{rr}
0 & \sigma_{r} \\
-\sigma_{r} & 0
\end{array}\right]+\varepsilon \mu\left[\begin{array}{cc}
\sigma_{r} & 0 \\
0 & \sigma_{r}
\end{array}\right],
$$

and $z$ is the column vector obtained by excluding $u_{r}$ and $v_{r}$ from $(u, v, \xi, \eta)$, and $M$ is the $(2 n-2) \times(2 n-2)$ constant matrix whose eigenvalues are not integer multiples of $\imath \sigma_{r}$. In our search for periodic solutions with period $T$ near $2 \pi /\left|\sigma_{r}\right|$, we are led to the periodicity condition

$$
\begin{aligned}
& w_{r}(T)-w_{r}(0)=\{\exp (L T)-I\} w_{r}(0)+O\left(\varepsilon^{2}\right)=0, \\
& z(T)-z(0)=\{\exp (M T)-I\} z(0)+O(\varepsilon)=0 .
\end{aligned}
$$

Without loss of generality, we may assume $\sigma_{r}>0$ in the following. By setting $T=2 \pi \sigma_{r}^{-1}(1+\varepsilon \delta)$ with a new parameter $\delta$ and restricting ourselves to the initial condition $u_{r}(0)=\sqrt{2}, v_{r}(0)=0$, this periodicity condition leads to the following so called "bifurcation equations"

$$
\begin{aligned}
& \Gamma_{1}=\frac{1}{2 \pi \varepsilon}\left\{w_{r}(T)-w_{r}(0)\right\}=\sqrt{2}\left[\begin{array}{c}
\mu \\
-\delta
\end{array}\right]+O(\varepsilon)=0, \\
& \Gamma_{2}=\left[\exp \left\{\left(2 \pi / \sigma_{r}\right) M\right\}-I\right] z(0)+O(\varepsilon)=0 .
\end{aligned}
$$


This can be considered as the implicit system for $\mu, \delta, z(0)$. For $\varepsilon=0$ this system has a unique solution $\mu=\delta=0$ and $z(0)=0$. Since the determinant of the Jacobian matrix $\partial\left(\Gamma_{1}, \Gamma_{2}\right) / \partial(\mu, \delta, z(0))$ does not vanish at this point, the bifurcation equations can be solved for $\mu, \delta, z(0)$ as functions of sufficiently small $\varepsilon$.

Thus we have determined the initial values $u_{r}(0), v_{r}(0)$ and $z(0)$ depending on the parameter $\varepsilon$, which gives a family of periodic solutions for (3.10), i. e., for (3.9). Obviously each periodic orbit belonging to this family intersects $\Sigma$ transversally at a point satisfying (3.6), and its primitive period is $2 \pi / \sigma_{r}+O\left(\varepsilon^{2}\right)$. Indeed, we have evidently $\partial H / \partial u_{r} \neq 0$ at the intersection point of each periodic orbit with $\Sigma$. This also implies that $\mu=0$ along these periodic solutions. Therefore this defines our desired periodic family.

Q.E.D.

ii) The Poncaré mappings assocıated to the Lıapunov's perıdic family.

We will investigate the Poincaré mapping in the coordinates after introducing the stretching transformation (3.1). We note that each periodic orbit given in Lemma 2 lies on a energy surface (3.7), which corresponds to $\hat{H}=\sigma_{r}+O\left(\varepsilon^{2}\right)=h$ with $\varepsilon$ fixed. We now define a $(2 n-2)$-dimensional manifold $\Sigma_{s}$ by $\Sigma_{\varepsilon}=\Sigma \cap$ $\{\hat{H}=h\}$. The points in $\Sigma_{\varepsilon}$ are parametrized by the $2 n-2$ variables $u_{1}, \cdots, u_{r-1}$, $v_{1}, \cdots, v_{r-1}$ and $\zeta$, with $v_{r}=0$ and $u_{r}$ being determined implicitly from the relation $\hat{H}=h=\sigma_{r}+O\left(\varepsilon^{2}\right)$. Then, in the coordinates after introducing the stretching transformation (3.1), we can define the Poincaré mapping $\varphi_{\varepsilon}$ near each periodic orbit given in Lemma 2 by following the solution of (3.3) from a point in $\Sigma_{\text {s }}$ with increasing $t$ to its next intersection with $\Sigma_{s}$.

In order to obtain this mapping, we first note that the system (3.3) can be written in the form

$$
\begin{aligned}
& \frac{d u_{k}}{d t}=\left(\sigma_{k}+\varepsilon^{2} \sum_{l=1}^{r} q_{k l} \tau_{l}\right) v_{k}+O\left(\varepsilon^{3}\right), \\
& \frac{d v_{k}}{d t}=\left(-\sigma_{k}-\varepsilon^{2} \sum_{l=1}^{r} q_{k l} \tau_{l}\right) u_{k}+O\left(\varepsilon^{3}\right), \quad(k=1, \cdots, r) \\
& \frac{d \zeta}{d t}=C \zeta+O(\varepsilon), \quad C=J_{s} B .
\end{aligned}
$$

Since $d \tau_{k} / d t=O\left(\varepsilon^{3}\right)$, by integrating this system we have

$$
\begin{aligned}
& u_{k}(t)=u_{k} \cos \left(t \Phi_{k}\right)+v_{k} \sin \left(t \Phi_{k}\right)+O\left(\varepsilon^{3}\right), \\
& v_{k}(t)=-u_{k} \sin \left(t \Phi_{k}\right)+v_{k} \cos \left(t \Phi_{k}\right)+O\left(\varepsilon^{3}\right), \\
& \Phi_{k}=\sigma_{k}+\varepsilon^{2} \sum_{l=1}^{r} q_{k l} \tau_{l}, \\
& \zeta(t)=\{\exp (t C)\} \zeta+O(\varepsilon),
\end{aligned}
$$

where we used $(u, v, \zeta)$ and $\tau_{l}$ in place of $(u(0), v(0), \zeta(0))$ and $\tau_{l}(0)$ respectively. The time when this solution intersects $\Sigma_{\varepsilon}$ first again is regarded as a function of these initial values $u, v, \zeta$ and $\varepsilon$. It is given by the form 


$$
T=\frac{2 \pi}{\Phi_{r}}+O\left(\varepsilon^{3}\right)=\frac{2 \pi}{\sigma_{r}}+\varepsilon^{2} \rho+O\left(\varepsilon^{3}\right), \quad \rho=\left(-2 \pi / \sigma_{r}^{2}\right) \sum_{l=1}^{r} q_{r l} \tau_{l} .
$$

Furthermore, by the relation $\hat{H}=h=\sigma_{r}+O\left(\varepsilon^{2}\right)$ we have

$$
\tau_{r}=\frac{1}{\sigma_{r}}\left(h-\sum_{k=1}^{r-1} \sigma_{k} \tau_{k}\right)+O\left(\varepsilon^{2}\right)=1-\frac{1}{\sigma_{r}} \sum_{k=1}^{r-1} \sigma_{k} \tau_{k}+O\left(\varepsilon^{2}\right),
$$

which eliminates $u_{r}$ with $v_{r}=0$ in $\Sigma_{\varepsilon}$. Therefore the Poincaré mapping $\varphi_{\varepsilon}:(u, v, \zeta)$ $\rightarrow\left(u^{(1)}, v^{(1)}, \zeta^{(1)}\right)=(u(T), v(T), \zeta(T))$ is given by the following form:

$$
\begin{aligned}
& u_{k}^{(1)}=u_{k} \cos \Psi_{k}+v_{k} \sin \Psi_{k}+O\left(\varepsilon^{3}\right), \\
& v_{k}^{(1)}=-u_{k} \sin \Psi_{k}+v_{k} \cos \Psi_{k}+O\left(\varepsilon^{3}\right), \quad(k=1, \cdots, r-1) \\
& \zeta^{(1)}=\exp \left\{\left(2 \pi / \sigma_{r}\right) C\right\} \zeta+O(\varepsilon), \quad C=J_{s} B,
\end{aligned}
$$

where

$$
\begin{aligned}
& \Psi_{k}=\frac{2 \pi}{\sigma_{r}}\left(\sigma_{k}+\varepsilon^{2} \alpha_{k}+\varepsilon^{2} \sum_{l=1}^{r-1} \beta_{k l} \tau_{l}\right) ; \\
& \alpha_{k}=q_{k r}-\frac{\sigma_{k}}{\sigma_{r}} q_{r r}, \quad \beta_{k l}=\left(q_{k l}-\frac{\sigma_{k}}{\sigma_{r}} q_{r l}\right)-\frac{\sigma_{l}}{\sigma_{r}}\left(q_{k r}-\frac{\sigma_{k}}{\sigma_{r}} q_{r r}\right) .
\end{aligned}
$$

Here the intersection of each periodic orbit given in Lemma 2 with $\Sigma_{\varepsilon}$ corresponds to the fixed point for this mapping. By the remark (iv) to Lemma 2, the transformation which takes each fixed point into the origin is of the form

$$
u_{k} \longrightarrow u_{k}+O(\varepsilon), \quad v_{k} \longrightarrow v_{k}+O(\varepsilon) \quad(k=1, \cdots, r-1), \quad \zeta \longrightarrow \zeta+O(\varepsilon) .
$$

We can easily see that the Poincaré mapping $\varphi_{\varepsilon}$ does not change its form (3.13) with (3.14) under this transformation. Hence the mapping (3.13) can be considered to be defined in a suitably large neighborhood of the origin with suffciently small $\varepsilon>0$, and to have the origin as a fixed point for any $\varepsilon>0$. In what follows, we call this mapping $\phi_{\varepsilon}$.

iii) Reduction to the $(r-1)$-dimensional torn.

Now we will seek for periodic points for the mapping $\phi_{\varepsilon}$. If we consider this mapping $\phi_{\varepsilon}$ in the original coordinates, which is given by

$$
\varepsilon u_{k} \longrightarrow \varepsilon u_{k}^{(1)}, \quad \varepsilon v_{k} \longrightarrow \varepsilon v_{k}^{(1)}(k=1, \cdots, r-1), \quad \varepsilon^{2} \zeta \longrightarrow \varepsilon^{2} \zeta^{(1)},
$$

then it is well known that this mapping (3.15) is exact-canonical. This implies that for any closed curve $\gamma$ in $\sum_{\varepsilon}$ the integral

$$
\varepsilon^{2} \int_{\gamma}^{r-1}\left(u_{k} d v_{k}-v_{k} d u_{k}\right)+\varepsilon^{4} \int_{\gamma} \sum_{k=1}^{s}\left(\xi_{k} d \eta_{k}-\eta_{k} d \xi_{k}\right)
$$

is invariant under the mapping $\phi_{\varepsilon}$.

We consider the $p$-th iterated $\phi_{\varepsilon}^{(p)}$ of $\phi_{\varepsilon}$, where $p=\left|\sigma_{r} / \sigma_{1}\right|$. Our aim is to find fixed points for $\phi_{\varepsilon}^{(p)}$, and it will be accomplished in two steps. The first step is to restrict ourselves to seeking fixed points for $\phi_{\varepsilon}^{(p)}$ on an $(r-1)$-torus. 
To this end, we first introduce the new coordinates $\left(\tau_{k}, \theta_{k}\right)$ in place of $\left(u_{k}, v_{k}\right)$ by

$$
u_{k}=\sqrt{2 \tau_{k}} \cos \theta_{k}, \quad v_{k}=\sqrt{2 \tau_{k}} \sin \theta_{k} \quad(k=1, \cdots, r-1),
$$

with $\zeta=\left(\xi_{1}, \cdots, \xi_{s}, \eta_{1}, \cdots, \eta_{s}\right)$ unchanged. Then the $p$-th iterated $\phi_{\varepsilon}^{(p)}$ of $\phi_{\varepsilon}$ is transformed into

$$
\begin{aligned}
& \tau_{k}^{(p)}=\tau_{k}+O\left(\varepsilon^{3}\right), \\
& \theta_{k}^{(p)}=\theta_{k}+p \Psi_{k}(\tau, \theta, \varepsilon)+O\left(\varepsilon^{3}\right), \quad(k=1, \cdots, r-1) \\
& \zeta^{(p)}=\exp \left\{\left(2 \pi / \sigma_{1}\right) C\right\} \zeta+O(\varepsilon) .
\end{aligned}
$$

We will solve the $2 n-r-1$ relations $\theta_{k}^{(p)} \equiv \theta_{k}(\bmod 2 \pi ; k=1, \cdots, r-1)$ and $\zeta^{(p)}=\zeta$ for $\tau_{1}, \cdots, \tau_{r-1}$ and $\zeta$ as an implicit system. Noting that

$$
\theta_{k}^{(p)}-\theta_{k} \equiv \varepsilon^{2} \frac{2 \pi p}{\sigma_{r}}\left(\alpha_{k}+\sum_{l=1}^{r-1} \beta_{k l} \tau_{l}\right)+O\left(\varepsilon^{3}\right),
$$

it suffices to solve the following $2 n-r-1$ equations:

$$
\begin{aligned}
& \Gamma_{k}=\alpha_{k}+\sum_{l=1}^{r-1} \beta_{k l} \tau_{l}+O(\varepsilon)=0 \quad(k=1, \cdots, r-1), \\
& \Gamma=\left[\exp \left\{\left(2 \pi / \sigma_{1}\right) C\right\}-I\right] \zeta+O(\varepsilon)=0 .
\end{aligned}
$$

For $\varepsilon=0$, (3.19) is satisfied with $\zeta=0$ and (3.18) leads to the linear equations for $\tau_{1}, \cdots, \tau_{r-1}$,

$$
\left(\tau_{1}, \cdots, \tau_{r-1}\right)\left(\beta_{k l}\right)=-\left(\alpha_{1}, \cdots, \alpha_{r-1}\right) .
$$

If $\left|\beta_{k l}\right| \neq 0$, by the Cramér's formula we have

$$
\tau_{k}=\frac{-1}{\left|\beta_{k l}\right|} \sum_{l=1}^{r-1} \tilde{\beta}_{l k} \alpha_{l} \quad(k=1, \cdots, r-1),
$$

where $\tilde{\beta}_{l_{k}}$ denotes the cofactor of the elements $\beta_{l_{k}}$ in the $(r-1) \times(r-1)$ matrix $\left(\beta_{l k}\right)$. Here we obtain the identıties

$$
|P|=-\sigma_{r}^{2}\left|\beta_{k l}\right|, \quad \sigma_{r}\left|Q_{k}\right|=-\sigma_{r}^{2} \sum_{l=1}^{r-1} \tilde{\beta}_{l k} \alpha_{l}
$$

from equivalent relations

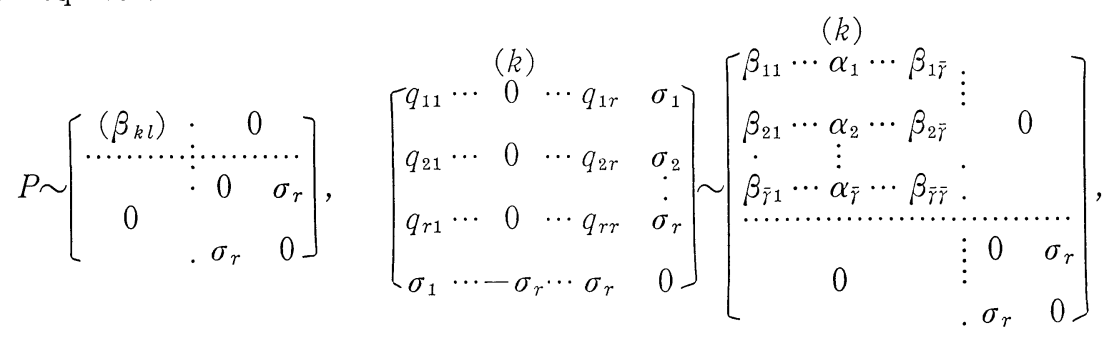

where $\bar{r}=r-1$. Hence, it follows from (3.20) together with (3.12) that 


$$
\tau_{k}=-\sigma_{r} \frac{\left|Q_{k}\right|}{|P|}>0 \quad(k=1, \cdots, r)
$$

under the assumption (2.6). Moreover, the Jacobian with respect to $\tau_{1}, \cdots, \tau_{r-1}$ and $\zeta$ does not vanish on the $(r-1)$-torus given by (3.21) and $\zeta=0$ under the assumption (2.6) with [A.2]. Therefore the implicit system of equations (3.18) and (3.19) can be solved for $\tau_{k}>0(k=1, \cdots, r-1)$ and $\zeta$ as functions of $\theta_{1}, \cdots$, $\theta_{r-1}$ and $\varepsilon$ with $\varepsilon=0$ corresponding to any point on this $(r-1)$-torus given by (3.21) and $\zeta=0$. For each $\varepsilon>0$, this solution defines an $(r-1)$-torus $\Lambda_{\varepsilon}$ in $\Sigma_{\varepsilon}$ given by

$$
\begin{aligned}
\Lambda_{\varepsilon}= & \left\{\tau_{k}=\tau_{k}(\theta, \varepsilon), k=1, \cdots, r-1, \zeta=\zeta(\theta, \varepsilon)\left(\theta=\left(\theta_{1}, \cdots, \theta_{r-1}\right)\right)\right. \\
& \left.\mid \theta_{k}^{(p)} \equiv \theta_{k}, k=1, \cdots, r-1, \zeta^{(p)}=\zeta\right\} .
\end{aligned}
$$

Thus the existence of fixed points for $\phi_{\varepsilon}^{(p)}$ in $\sum_{\varepsilon}$ is reduced to the existence of points $\theta$ on $\Lambda_{\varepsilon}$ where we have $\tau_{k}^{(p)}=\tau_{k}(k=1, \cdots, r-1)$. Finally we note that the fixed points define our desired periodic orbits with primitive periods near $2 \pi /\left|\sigma_{1}\right|$ since there exist no fixed point for $\phi_{\varepsilon}^{(m)}(m=1, \cdots, p-1)$ on the torus $\Lambda_{\varepsilon}$.

iv) The existence of fixed points for $\phi_{\varepsilon}^{(p)}$ on the torus $\Lambda_{\varepsilon}$.

Finally we discuss the existence of fixed points for $\phi_{\varepsilon}^{(p)}$ on the $(r-1)$-torus $\Lambda_{\varepsilon}$, namely that of points $\theta$ on $\Lambda_{\varepsilon}$ such that $\tau_{k}^{(p)}=\tau_{k}(k=1, \cdots, r-1)$. Since not only the integral (3.16) but also the second integral of (3.16) is invariant under the mapping $\phi_{\varepsilon}^{(p)}$ restricted to $\Lambda_{\varepsilon}$, it follows that for any closed curve $\gamma$ on $\Lambda_{\varepsilon}$,

$$
\int_{\gamma=1}^{r-1}\left(u_{k} d v_{k}-v_{k} d u_{k}\right)=2 \int_{\gamma} \sum_{k=1}^{r-1} \tau_{k} d \theta_{k}
$$

is invariant under the mapping $\phi_{\varepsilon}^{(p)}$. In other words, we have

$$
\int_{\gamma} \sum_{k=1}^{r-1}\left(\tau_{k}^{(p)} d \theta_{k}^{(p)}-\tau_{k} d \theta_{k}\right)=0
$$

for any closed curve $\gamma$ on $\Lambda_{\varepsilon}$. Here, noting that $d \theta_{k}^{(p)}=d \theta_{k}$ on $\Lambda_{\varepsilon}$ since $\theta_{k}^{(p)} \equiv$ $\theta_{k}(k=1, \cdots, r-1)$, we have

$$
\int_{\gamma}^{r-1}\left(\tau_{k}^{(p)}-\tau_{k}\right) d \theta_{k}=0
$$

for any closed curve $\gamma$ on $\Lambda_{\varepsilon}$. Hence the integral

$$
F(\theta)=\int_{0}^{\theta} \sum_{k=1}^{r-1}\left(\tau_{k}^{(p)}-\tau_{k}\right) d \theta_{k}, \quad \theta=\left(\theta_{1}, \cdots, \theta_{r-1}\right),
$$

is independent of the choice of the path and therefore defines a smooth function on the torus with period $2 \pi$ in $\theta_{1}, \cdots, \theta_{r-1}$. Further this function $F(\theta)=$ $F(\theta, \varepsilon)$ satisfies

$$
\tau_{k}^{(p)}-\tau_{k}=\frac{\partial F}{\partial \theta_{k}} \quad(k=1, \cdots, r-1) .
$$


Thus the existence of our desired fixed points is reduced to the existence of critical points of $F(\theta)$ on the torus $\Lambda_{\varepsilon}$. As is well known, a smooth function on an $m$-dimensional torus has at least $2^{m}$ critical points, counting multiplicities, of which at least $m+1$ are geometrically distinct (see e.g. [11]). Therefore the function $F(\theta)$ has at least $r$ geometrically distinct critical points on the torus $\Lambda_{\varepsilon}$. Hence we have proved that there exist at least $r$ geometrically distinct fixed points on this torus, which provides our desired periodic orbits. The proof of the theorem is now completed.

Q.E.D.

\section{Remarks.}

In the preceding proof, it was essential to obtain a one-parameter family of canonical mappings of the form (3.13) with (3.14) near the Liapunov's periodic family with primitive periods near $2 \pi /\left|\sigma_{r}\right|$. To this end, Lemma 2 is important. However, if some eigenvalue $\lambda_{\jmath}=\imath \sigma_{\jmath}(\jmath=2, \cdots, r-1)$ other than $\lambda_{r}$ satisfies the non-resonance condition, i. e., none of the quotients $\lambda_{k} / \lambda_{j}(k=1, \cdots, r, k \neq j)$ is an integer, the same assertion as in Lemma 2 holds for the Liapunov's periodic family corresponding to this eigenvalue $\lambda_{3}$. Then we shall obtain a one-parameter family of Poincaré mappings of the same form as (3.13) with (3.14), and each of them can be considered to be defined in a neighborhood of the origin, which is a fixed point corresponding to the Liapunov's periodic orbit with primitive period near $2 \pi /\left|\sigma_{\jmath}\right|$. In the same way as in the preceding proof, we can discuss the existence of fixed points for the $p$-th iterated $\phi_{\varepsilon}^{(p)}$ of these mappings $\phi_{\varepsilon}$ with $p=\sigma_{j} / \sigma_{1}$, which correspond to the periodic orbits whose primitive periods are close to $2 \pi /\left|\sigma_{1}\right|$. Hence we shall have the same assertion as in theorem $\mathrm{A}$, in other words, we shall have another sufficient condition for the existence of periodic orbits with primitive periods near $2 \pi /\left|\sigma_{1}\right|$ under [A.1] and [A.2]. Indeed, we can have more general assertion as follows:

Let us impose the following conditions on the eigenvalues $\pm \lambda_{k}(k=1, \cdots, n)$ of $J S$;

[B.1] $\pm \lambda_{k}= \pm \imath \sigma_{k}(k=1, \cdots, r)$ are $r$ pairs of eigenvalues all of which are integer multiples of a purely imaginary number $\lambda_{0}=\imath \sigma_{0}\left(\sigma_{0}>0\right)$ such that

and

$$
\left(\left|\sigma_{1} / \sigma_{0}\right|,\left|\sigma_{2} / \sigma_{0}\right|, \cdots,\left|\sigma_{r} / \sigma_{0}\right|\right)=1
$$

$$
\sum_{k=1}^{r} \jmath_{k} \sigma_{k} \neq 0
$$

for all integer valued vectors $\left(\jmath_{1}, \cdots, \jmath_{r}\right)$ with $1 \leqq \sum_{k=1}^{r}\left|\jmath_{k}\right| \leqq 4$. Here $\left(m_{1}, m_{2}, \cdots, m_{r}\right)$ stands for the greatest common measure among the $r$ positive integers $m_{k}=\left|\sigma_{k} / \sigma_{0}\right|(k=1, \cdots, r)$.

[B.2] no $\pm \lambda_{k+r}(k=1, \cdots, s)$ is an integer multiple of $\lambda_{0}$, where $s=n-r$.

Then we have the Lemma 1 under [B.1] and [B.2] instead of [A.1] and [A.2], 
and we can prove

Theorem B. Consider the Hamiltonian system (1.1) near the origin. Let the eigenvalues of JS satisfy [B.1] and [B.2], and suppose that $\lambda_{j}(1 \leqq j \leqq r)$ satisfies the non-resonance condition, i.e., none of the quotients $\lambda_{k} / \lambda_{j}(k=1, \cdots, r . k \neq j)$ is an integer. Assume in the normal form (2.1) that

$$
\left(\sigma_{\jmath}|P|\right) \cdot\left|Q_{k}\right|<0 \quad(k=1, \cdots, r) .
$$

Then, on each energy surface $H=\sigma_{j} \varepsilon^{2}$ with sufficiently small $\varepsilon>0$, there exist at least $r$ periodic orbits for (1.1) with primitvve periods near $2 \pi / \sigma_{0}$ in a $O(\varepsilon)$ neighborhood of the periodic orbit belonging to the Liapunov's periodic family whose primitive period is close to $2 \pi /\left|\sigma_{\jmath}\right|$.

The proof of this theorem is exactly the same as that of theorem $A$, and then we omit the proof. We note here that theorem B includes theorem $A$ as a special case when $\lambda_{0}=\lambda_{1}$ and $\lambda_{j}=\lambda_{r}$.

We finally remark the connection of the above result with that of [7]. The periodic orbits whose existence is guaranteed by theorems A and B are near the equilibrium. We have already obtained a sufficient condition for the existence of periodic orbits near the equilibrium whose primitive periods are close to $2 \pi /\left|\sigma_{1}\right|$ under [A.1] and [A.2] (see (3.2) in [7]). It is given in terms of the normal form (2.1) by

$$
q_{k l}-\frac{\sigma_{k}}{\sigma_{1}} q_{11} \neq 0 \quad(k=2, \cdots, r) .
$$

If none of the $r-1$ relations in (4.2) is satisfied, i. e., $q_{k 1}-\sigma_{k} / \sigma_{1} \cdot q_{11}=0$ ( $k=$ $2, \cdots, r)$, then we have $\left|Q_{k}\right|=0(k=2, \cdots, r)$, that is, the condition (2.6) is not satisfied. Therefore we see that the condition (2.6) includes (4.2) especially when $r=2$. However, when $r \geqq 3$, the conditions (2.6) and (4.2) are independent. In fact, for example, let us consider the case when $n=r=3$ in [A.1] and [A.2], and take the Hamiltonian function $H(u, v)$ of the normal form $(2.1)$ such that

$$
q_{11}=q_{22}=q_{33}=1, \quad q_{21}=\sigma_{2} / \sigma_{1}, \quad q_{31}=\rho>0, \quad q_{32}=0
$$

together with $\sigma_{1}>0, \sigma_{2}<0$ and $\sigma_{3}>0$. Here we note that $q_{k l}=q_{l k}$ and take $\rho>0$ suitably small. Then we have $|P|>0$ and $\left|Q_{k}\right|<0 \quad(k=1,2,3)$. Therefore the condition (2.6) is satisfied, but (4.2) is not satisfied. Conversely, we can also make examples where (4.2) is satisfied but (2.6) is not.

\section{REFERENCES}

[1] V.I. Arnold, Mathematical Methods of Classical Mechanics, Springer-Verlag, 1978.

[2] G. D. Birkhoff, Dynamical Systems, American Mathematical Society, 1966.

[3] G. D. BirkHofF, Une généralisation à $n$-dimensions du dernier théorème de géométrie de Poincaré, Compt. Rend. Acad. Sci. 192 (1931), 196-198. 
[4] G. D. BiRKHofF AND D.C. LEwis, On the periodic motions near a given periodic motion of a dynamical system, Ann. Mat. Pura Appl. 12 (1933), 117-133.

[5] S. M. GRAFF, On the conservation of hyperbolic invariant tori for Hamiltonian systems, J. Differential Equations 15 (1974), 1-69.

[6] T.C. HARRIs, Periodic solutions of arbitrary long periods in Hamiltonian systems, J. Differential Equations 4 (1968), 131-141.

[7] H. ITo, Long periodic solutions near an equilibrium in general resonance cases, Kodai Math. J. 3 (1980), 442-456.

[8] K. R. Meyer and J. I. Palmore, A new class of periodic solutions in the restricted three body problem, J. Differential Equations 8 (1970), 264-276.

[9] J. Moser, Proof of a generalized form of a fixed point theorem due to G. D. Birkhoff, in Geometry and Topology, Springer Lecture Notes in Math., No. 597, Proceedings of the Selcool held at IMPA, Rio de Janeiro, 1976, Springer-Verlag, 1977, 464-494.

[10] D. S. Schmid, Hopf's bifurcation theorem and the center theorem of Liapunov with resonance cases, J. Math. Anal. Appl. 63 (1978), 354-370.

[11] J. T. Schwartz, Nonlinear Functional Analysis, Gordon and Breach, 1969.

[12] C. L. Siegel and J. K. Moser, Lecture on Celestial Mechanics, Springer-Verlag, 1971.

Department of Mathematics

Tokyo Institute of Technology 